\section{Scientific bodies must take own action on emissions}

SIR - Many of the world's most reputable and best-placed scientific organizations, including the American Association for the Advancement of Science, the Royal Society, the American Geophysical Union and the American Meteorological Society, have released strong and unequivocal statements regarding the dangers the world's population faces as anthropogenic climate change gains pace. Although such statements are effective in informing public opinion and thereby influencing policy on this important issue, they are not the most powerful means available.

A more potent approach would be for scientific organizations to make ambitious, high-profile moves to reduce their own contributions to climate change. Such activity could generate significant publicity and demonstrate that the organizations are taking the threat of climate change seriously. They would send a louder, clearer message that emissions reduction should be a priority.

Such moves, although necessarily bold, should not impair the organizations' abilities to achieve their primary aims. Rather, they should publicly demonstrate that reductions in any organization's environmental impact need not reduce its effectiveness. One example would be the more widespread inclusion of video-conferencing facilities in oral sessions at scientific meetings. Another could be the introduction of 'virtual poster sessions' with live audio connections.

If well-implemented, such measures would actually increase the effectiveness of a meeting while reducing its environmental impact. In particular, those who would otherwise not attend could now participate, which would lead to an increase both in the dissemination of research findings and in the interaction between members of the organization.

Andrew Biggin

Paleomagnetic Laboratory Fort Hoofddijk,

Faculteit Geowetenschappen,

Universiteit Utrecht, Budapestlaan 17,

3584 CD Utrecht, the Netherlands

\section{Bush has not obstructed environmental protection}

SIR - Your recent Editorial 'An end in sight' (Nature 447, 886; 2007), is completely off base in saying that the US Environmental Protection Agency's mission has been obstructed by the Bush administration. In fact, the EPA's enforcement programme is stronger than ever. The agency was also voted one of the ten best places to work in the federal government, according to the Partnership for Public Service, an organization based in Washington DC that aims to establish the federal government as an attractive employer for top workers.

During the past three years, EPA's enforcement programme has prevented nearly 1.3 million tonnes of pollution from entering the environment. Its enforcement actions have required defendants to invest $\$ 19.7$ billion to reduce pollution at facilities, clean up spills, install new pollution-control equipment and clean up contaminated soil or water. And thanks to the efforts of a revitalized criminal enforcement programme in 2006, offenders will serve a total of 154 years in jail and pay almost $\$ 43$ million in fines, as well as another $\$ 29$ million for environmental projects imposed as part of their sentences.

Granta Nakayama

Office of Enforcement and Compliance

Assurance, US Environmental Protection Agency, Washington, DC 20460, USA

\section{Chemical reaction to the many-worlds hypothesis}

SIR - Your Editorial 'Parallel worlds galore', celebrating the fiftieth anniversary of the multiverse hypothesis (Nature 448, 1; 2007), was a timely and stimulating reminder that the interaction of science and fiction sometimes leads to facts, or at least to scientific implications. But in your two News Features on the topic (Nature 448,15-17 and 18-21;2007), I wonder why chemistry was largely ignored, as this discipline offers excellent scientific platforms for sciencefiction narrative. Likewise, fiction holds lessons for chemistry.

Chemists have always wondered whether life in a parallel universe could actually be based on atoms other than carbon. Perhaps that thinking inspired Lewis Carroll's Alice to call into question whether milk could be different in the universe behind the mirror in Through the Looking Glass.

Although Kurt Vonnegut's Galapagos is quoted as a great example of science fiction in biology, his 1963 novel Cat's Cradle has lessons for science. Here, the protagonist, Felix Hoenikker, creates a solid form of water called ice-nine - a narrative probably influenced by the work of Nobel laureate Irving Langmuir. At least 12 forms of ice have now been reported, including ice IX though this is unlike the fictional ice-nine. Cat's Cradle also inspired interest in what seemed to be an extraordinary form of water with anomalous properties, known as 'polywater'. This Russian discovery may have had its roots in papers published during the late 1920s on the vapour pressure of water and other liquids in small capillaries (see, for example, J. L. Shereshefsky J. Am. Chem.
Soc. 50, 2966-2980 and 2980-2985; 1928). But the research caused much excitement when it was presented in the West in 1966, and it became widely accepted until proved, a few years later, to be fallacious.

It seems appropriate, therefore, to consider chemistry as a tool for uncovering facts that either inspire or imitate fiction. What is really astonishing, to a chemist, about a parallel world coexisting with our own is not its existence in itself, but rather the matter we could find there.

Pedro Cintas

Department of Organic and Inorganic

Chemistry, University of Extremadura,

E-06071 Badajoz, Spain

\section{International research may leave women adrift}

SIR - The difficulties facing women and under-represented minorities in science and engineering are highlighted in your Naturejobs Special Reports 'Beyond the glass ceiling' and 'Closing the gender gap' (Nature 448, 98-100 and 101-102; 2007). But women from developing countries who work abroad face additional problems, which are not addressed by efforts to help either women or minorities.

During our studies and beyond, we face financial troubles, assumptions of ineptitude by faculty members, harassment and lack of mentoring. In a survey I conducted among 13 women who graduated from the Indian Institutes of Technology and went to the United States, several reported problems with supervisors, including harassment or having to switch supervisors; two of them eventually left research because of lack of support. On top of that, the stress caused by problems with elderly family members at home can be enormous and is not always understood by people in Western societies, whose family ties and responsibilities may differ. Visa problems are also not unusual.

For those of us who return to our home countries, there are more hurdles to clear. Institutions in many developing countries do not open their doors easily to women, even if they actively seek out and recruit men who have studied abroad. Often it is hard to find a position that is appropriate for our education and experience. And the styles of working abroad and at home can be very different.

To make the best use of our talents and training - whether in the United States, Europe or elsewhere - a full survey of our concerns should be conducted and used to frame new policies and instil change within academic culture.

Asha Gopinathan

GenSci-e-Tech, E 2 Sree Vilas Lane, Kaudiar, Trivandrum 695003, Kerala, India

dendron.15@gmail.com 